\title{
Guidelines
}

\section{Comparison of the European and Japanese Guidelines for the Acute Management of Intracerebral Hemorrhage}

\author{
Kazunori Toyoda ${ }^{a}$ Thorsten Steiner $^{b, c}$ Corina Epple ${ }^{c}$ Rolf Kern ${ }^{d}$ \\ Masao Nagayama $^{e}$ Yukito Shinohara $^{f}$ Michael G. Hennerici ${ }^{d}$ \\ ${ }^{a}$ Department of Cerebrovascular Medicine, National Cerebral and Cardiovascular Center, Suita, Osaka, \\ Japan; ' Department of Neurology, Klinikum Frankfurt Höchst, Frankfurt, ' Department of Neurology, University of \\ Heidelberg Hospital, Heidelberg, and dDepartment of Neurology, UMM, University of Heidelberg, Mannheim,

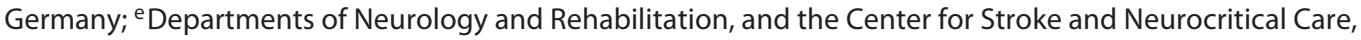 \\ International University of Health and Welfare Atami Hospital, Atami, and fDepartment of Neurology, Federation of \\ National Public Service Personnel Mutual Aid Associations Tachikawa Hospital, Tokyo, Japan
}

\section{Key Words}

Acute stroke treatment - European stroke guidelines ·

Evidence-based medicine - Hemorrhage - Japanese stroke guidelines · Randomized clinical trials · Review · Stroke prevention

\footnotetext{
Abstract

Background: Different aspects of acute stroke management and strategies for stroke prevention derive from two viewpoints: specific traditional and historical backgrounds and evidence-based medicine from modern randomized controlled trials (RCTs), meta-analysis and authorized clinical practice guidelines (GLs). Regarding intracerebral hemorrhage (ICH), Cerebrovascular Diseases published the 2006 European stroke initiative recommendations for the management of $\mathrm{ICH}$. In 2009, the revised Japanese GLs for the management of stroke, including that of $\mathrm{ICH}$, appeared in Japanese. Whereas GLs for the prevention and treatment of ischemic stroke were presented in detail, recommendations
}

\section{KARGER}

E-Mail karger@karger.com www.karger.com/ced with regard to ICH are relatively rare both in Japan and Europe. Methods: Since 2011, the authors have met repeatedly and have compared the latest versions of published European and Japanese GLs for ischemic and hemorrhagic strokes. Many aspects have only been addressed in one but left out in the other GLs, which consequently founded the basis for the comparison. Classification of evidence levels and recommendation grades defined by the individual committees differed between both original GLs. Results: Aspects of major importance were similar and hence did not need extensive interpretation, mostly due to a lack of evidence from appropriate RCTs worldwide. The target level to which systolic blood pressure should be lowered is quite high; $<170 \mathrm{~mm} \mathrm{Hg}$ for patients with known hypertension in Europe and $<180 \mathrm{~mm} \mathrm{Hg}$ in Japan. The results of ongoing clinical trials are awaited for the optimal target level and optimal medications. Concerning $\mathrm{ICH}$ associated with oral an-

\section{K.T., T.S. and C.E. contributed equally to this work.}

(c) 2013 S. Karger AG, Basel

$1015-9770 / 13 / 0355-0419 \$ 38.00 / 0$
Dr. Kazunori Toyoda

Department of Cerebrovascular Medicine National Cerebral and Cardiovascular Center 5-7-1 Fujishirodai, Suita, Osaka 565-8565 (Japan)

E-Mail toyoda@ncvc.go.jp 
ticoagulant therapy, both guidelines give similar recommendations, namely that anticoagulation should be discontinuedand the international normalized ratio of prothrombin time should be normalized with prothrombin complex concentrate or fresh-frozen plasma and additional vitamin $\mathrm{K}$. Patients with ICH were treated surgically, often based on individual decisions - more frequently in Japan, depending on the association with hypertension. Patients with large or intraventricular bleedings were only treated if a life-saving performance was considered, irrespective of the neurological outcome. Infra- and supratentorial differences were similarly addressed in both GLs. Conclusion: This brief survey - when compared with the lengthy original recommendations - provides a stimulating basis for an extended interest among Japanese and European stroke clinicians to learn from their individual experiences and to strengthen efforts for joint cooperation in treating and preventing stroke all around the globe.

Copyright $\odot 2013$ S. Karger AG, Basel

Intracerebral hemorrhage (ICH) accounts for $10-17 \%$ of all strokes. The incidence of $\mathrm{ICH}$ is influenced by racial factors and was found to be higher in Blacks, Hispanics and Asians compared to the white population. In particular, Asian ethnic origin is a possible risk factor for intracranial hemorrhage. In 1965, Japan had the highest mortality of stroke worldwide with the mortality rate of $\mathrm{ICH}$ being quite high among all stroke types. A recent metaanalysis indicates that the incidence of ICH in the general Asian population was 2-fold higher than that in the white population [1].

We compared the European Stroke Initiative (EUSI) Recommendations for the management of ICH (2006) [2] and the chapters of ICH in the Japanese guidelines for the management of stroke (2009) [3]. Japanese guidelines are more up to date and thus included trials that were not available when EUSI guidelines were written. In the following, we try to present differences and similarities of both guidelines and worked out which recommendations lacked in both guidelines.

\section{Primary Prevention and Treatment of Risk Factors}

The EUSI guidelines give no recommendations for primary prevention, but give some evidence concerning different risk factors (table 1). Arterial hypertension is mentioned as the most common risk factor for sponta- neous ICH. The EUSI Writing Committee describes that the role of hypertension and the beneficial effect of antihypertensive treatment with regard to the risk of ICH were verified in PROGRESS (Perindopril Protection against Recurrent Stroke Study) [4], which showed that the relative risk of ICH was reduced by $50 \%$ in comparison with the placebo-treated group after a 4-year follow-up, but give no recommendation for treatment as primary prevention. In the Japanese guidelines, hypertension is regarded as the greatest risk factor for ICH and ischemic stroke. There is a positive linear correlation between blood pressure levels and the risk of stroke: the higher the blood pressure, the higher the risk of stroke [5]. Thus, medical antihypertensive treatment was strongly recommended as primary prevention [6].

Concerning hypercholesterolemia, the EUSI recommendations mention that hypercholesterolemia is associated with a lower risk of $\mathrm{ICH}$, but treatment with statins did not increase the risk of ICH. The risk of hemorrhagic stroke, including $\mathrm{ICH}$, is higher in smokers, but no recommendation of nonsmoking as a primary prevention is verbalized. An increased body mass index, which was found to be correlated with an increase in intraventricular hemorrhage (IVH) volume, or alcohol consumption are also considered risk factors for ICH. The Japanese guidelines recommend consumption of a moderate amount of vegetables and fruits every day, whereas no dietary advice was found in the EUSI guidelines.

For secondary prevention after ICH, EUSI guidelines give recommendations for lowering blood pressure with a diuretic and angiotensin-converting enzyme inhibitor (level A), excessive alcohol should be discouraged (class IV), persons with elevated body mass index should take a weight-reducing diet (despite lack of evidence) and smokers should quit smoking (class IV evidence).

\section{Acute Blood Pressure Management}

There is no randomized controlled trial comparing the severity and prognosis of patients with acute hypertension and ICH with/without blood pressure-lowering treatment (table 2). Further clinical studies need to be conducted in the future to determine the optimal target level to which blood pressure should be lowered from the perspective of clinical prognosis. The target level in the Japanese guidelines is quite high (systolic blood pressure $<180$ $\mathrm{mm} \mathrm{Hg}$ ) and differs much from the major expert opinions in the nationwide survey [7], where most experts re- 
Table 1. Primary prevention and treatment of risk factors

European guidelines 2006 (EUSI)

Hypertension

An important role with regard to the risk of $\mathrm{ICH}$.

Treatment of hypertension has a beneficial effect (level A).

\section{Hypercholesterolemia}

Associated with a lower risk of ICH; however, treatment with statins does not increase the risk of ICH (level B).

Smoking is a risk factor for ICH (level B).
Japanese guidelines 2009

Treating hypertension is the most vital step to reduce the risk of ICH (grade A).

It has generally been agreed that its underlying hepatic disease and coexisting hypertension should be treated.

Lowering serum cholesterol levels with statins does not increase the incidence of $\mathrm{ICH}$, but some data have implied that intervention for stroke patients may increase the recurrence of ICH (grade B).

People should be encouraged to take a moderate amount of vegetables and fruits every day (grade B).

\section{Alcohol consumption}

Several studies document an increased risk of ICH in relation to alcohol consumption. Spontaneous ICH can probably also be triggered by binge drinking (level C).

Heavy alcohol consumption leading to abnormal blood $\gamma$-GTP levels should be discouraged (grade B).

We recommend the very careful consideration of an appropriate dose of each antithrombotic drug and its dual medications that are required to control concurrent hypertension (grade B).

A variety of illicit drugs are known to cause ICH

(amphetamines, cocaine and phenylpropanolamine).

Table 2. Acute blood pressure (BP) management

European guidelines 2006 (EUSI)

Known hypertension (HPT)

If SBP $>180 \mathrm{~mm} \mathrm{Hg}$ or DBP $>105 \mathrm{~mm} \mathrm{Hg}$

$\rightarrow \mathrm{BP}<170 / 100 \mathrm{~mm} \mathrm{Hg}$ or MAP $<125 \mathrm{~mm} \mathrm{Hg}$ (class IV, level C)

Unknown history of HPT

If SBP $>160 \mathrm{~mm} \mathrm{Hg}$ or DBP $>95 \mathrm{~mm} \mathrm{Hg}$

$\rightarrow \mathrm{BP}<150 / 90 \mathrm{~mm} \mathrm{Hg}$ or MAP $<100 \mathrm{~mm} \mathrm{Hg}$ (class IV, level C)
Japanese guidelines 2009

maintain SBP $<180 \mathrm{~mm} \mathrm{Hg}$ or MAP $<130 \mathrm{~mm} \mathrm{Hg}$

(grade $\mathrm{C} 1$ )

Decrease pressure not more than $20 \%$ of MAP on admission (class IV, level C)

When ICP elevated (nitroprusside contraindicated)

$\rightarrow$ adapt thresholds to cerebral perfusion pressure $>70$ (class IV, level C)

Intravenous drugs recommended for better controllability (GCP)

no special hypotensive drug recommended, careful use of vasodilators (nitrates), because they induce brain HPT (grade C1)

$\mathrm{SBP}=$ Systolic blood pressure; $\mathrm{DBP}=$ diastolic $\mathrm{BP} ; \mathrm{MAP}=$ mean arterial pressure; $\mathrm{HPT}=$ hypertension.

when performing surgical treatment:

$\rightarrow$ more aggressive BP lowering (grade C1) garded $<140,<150$ or $<160 \mathrm{~mm} \mathrm{Hg}$ as a target systolic
blood pressure partly based on results of small domestic
studies $[8,9]$. The EUSI guidelines recommend target lev-
els and maximum levels of blood pressure for known and
unknown hypertension, and recommend an adaptation

garded $<140,<150$ or $<160 \mathrm{~mm} \mathrm{Hg}$ as a target systolic
blood pressure partly based on results of small domestic
studies $[8,9]$. The EUSI guidelines recommend target lev-
els and maximum levels of blood pressure for known and
unknown hypertension, and recommend an adaptation

garded $<140,<150$ or $<160 \mathrm{~mm} \mathrm{Hg}$ as a target systolic
blood pressure partly based on results of small domestic
studies $[8,9]$. The EUSI guidelines recommend target lev-
els and maximum levels of blood pressure for known and
unknown hypertension, and recommend an adaptation

garded $<140,<150$ or $<160 \mathrm{~mm} \mathrm{Hg}$ as a target systolic
blood pressure partly based on results of small domestic
studies $[8,9]$. The EUSI guidelines recommend target lev-
els and maximum levels of blood pressure for known and
unknown hypertension, and recommend an adaptation

garded $<140,<150$ or $<160 \mathrm{~mm} \mathrm{Hg}$ as a target systolic
blood pressure partly based on results of small domestic
studies $[8,9]$. The EUSI guidelines recommend target lev-
els and maximum levels of blood pressure for known and
unknown hypertension, and recommend an adaptation

Comparison of Stroke Guidelines: Acute

Management of Intracerebral Hemorrhage in case of elevated intracranial pressure (ICP). Routine blood pressure lowering is not recommended.

Furthermore, different medications are mentioned in the EUSI guidelines, especially intravenous drugs due to their better controllability. EUSI guidelines recommend 
a careful use of calcium antagonists because of their rapid and excessive hypotensive effect. In the Japanese guidelines, the use of calcium antagonists (e.g. nicardipine) is contraindicated in patients with hyperacute $\mathrm{ICH}$, because of vasodilating effects and possible antiplatelet actions of calcium antagonists; however, direct evidence for this is lacking. In the above-mentioned nationwide survey, nicardipine was the most popular agent for acute ICH management [7]. In 2011, the Japanese government finally ordered the pharmaceutical makers of nicardipine to revise the label. In the new label, description of contraindication for ICH was abolished. In 2012, several Asian nations similarly revised the label of nicardipine.

The EUSI or Japanese guidelines did not consider the results of ATACH (Antihypertensive Treatment of Acute Cerebral Hemorrhage) [10] and INTERACT (Intensive Blood Pressure Reduction in Acute Cerebral Hemorrhage Trial) [11], although the newer American Heart Association/American Stroke Association guidelines [12] advocate that lowering systolic blood pressure to $140 \mathrm{~mm} \mathrm{Hg}$ is probably safe based on the results of INTERACT [11]. The results of both INTERACT 2 [13] and ATACH II [14] are awaited, and might further answer the question of whether there are other benefits of early systolic blood pressure reduction in patients with $\mathrm{ICH}$, and provide clearer evidence for the target blood pressure and the choice of drugs. However, it seems reasonable to lower blood pressure cautiously appreciating a slight effect of antihypertensives on hematoma expansion reduction in an overall weak or equivocal context of evidence and considering that autoregulation is often preserved in the acute phase (as opposed to the postacute phase) of ICH and furthermore aggressive lowering of blood pressure theoretically carries the risk of cerebral ischemia in hypertensive patients.

\section{Acute Hemostatic Management}

EUSI guidelines do not recommend the use of hemostatic agents (as tranexamic acid, e-aminocaproic acid and aprotinin) to control bleeding, because no clinical efficacy has been demonstrated thus far (table 3). Japanese guidelines recommend blood products such as platelets, prothrombin complex concentrate (PCC) or fresh-frozen plasma (FFP) in hypertensive ICH only in case of concurrent abnormal blood coagulation or platelet counts, though such products have been rarely in clinical use as hemostatic agents unless patients were anticoagulant users in Japan.

Concerning ICH associated with oral anticoagulant therapy, both guidelines give similar recommendations, whereas Japanese guidelines prefer PCC rather than FFP (although PCC is not covered by the Japanese health insurance). Current questions arise on feasibility, safety and efficacy of prothrombin-dependent coagulation factors in concentrated PCC versus unconcentrated FFP versus single factors like recombinant coagulation factor VIIa. The current information on these questions is inconsistent, and any conclusion drawn towards the preference of one of these coagulants is premature. Time to reversal of the international normalized ratio (INR) seems to be the most important determinant, and minimizing delays in drug administration should have highest priority, as recommended in both guidelines. Initial dosages of PCC for the reversal are different between both guidelines: $10-30$ (or 10-50) U/kg in the EUSI recommendation and $500 \mathrm{U}$ regardless of body weight in the Japanese one.

There is a considerable dilemma concerning when to restart anticoagulant therapy following ICH. The optimum timing of the resumption of anticoagulation is a crucial issue with conflicting evidence. EUSI recommended to restart not earlier than 10-14 days after the index bleeding but to check indication first. The Japanese guidelines recommend use of heparin after normalization of INR in patients with an elevated risk for embolism, but do not mention the timing of heparinization. Among the respondents in the nationwide survey, the timing to restart anticoagulation varied greatly: within 4 days in $7 \%$, $5-7$ days in $21 \%, 8-14$ days in $25 \%, 15-28$ days in $28 \%$ and 29 days or later in $18 \%$ [15].

The new direct oral anticoagulants (DOAC) as dabigatran, rivaroxaban and apixaban seem to be a safer and more convenient replacement for warfarin. Due to fewer drug and food interaction, easier handling and a more favorable risk-benefit profile compared with warfarin, it is conceivable that the number of patients treated with DOAC will increase. The optimal management of DOAC-associated ICH is unknown and no specific antidote is available. For this reason, physicians are faced with new challenges, especially in emergency situations, and questions arise on how to manage emergency situations like the indication for thrombolysis in acute stroke, and the management of intracranial or gastrointestinal bleedings. In the future, guidelines have to give recommendations how to manage DOAC-associated hemorrhages, but data are still limited. 
Table 3. Acute hemostatic management

European guidelines 2006 (EUSI)

For non-antithrombotic users

Pending further data on efficacy and safety, recombinant factor VIIa should not be used outside of a phase III trial.

A phase III trial is needed to confirm the beneficial effect of recombinant factor VIIa in ICH (class IV, level B, obsolete).
Japanese guidelines 2009

Hypertensive ICH + normal coagulation

$\rightarrow$ Blood products are not recommendable (grade C2).

Hypertensive ICH + abnormal platelets or blood coagulation system + bleeding tendency

$\rightarrow$ consider blood products such as platelets, PCC and FFP (grade $\mathrm{C} 1$ ).

There is no adequate scientific evidence to support the use of capillary stabilizers or antiplasmin agents for the treatment of acute ICH (grade C1).

ICH during oral anticoagulant treatment (OAT) such as warfarin INR $>1.4$

$\rightarrow$ OAT should be discontinued and the INR should be normalized with PCC or FFP.

Intravenous vitamin $\mathrm{K}$ should be added (class IV).
Stop warfarin and normalize the prothrombin time (PT) INR to $\leq 1.35$ using vitamin $\mathrm{K}$ and blood products as soon as possible (grade $\mathrm{B}$ ).

Use of PCC (not covered by Japanese health insurance) is recommended rather than FFP (grade B).

With high risk or recurrent cerebral embolism

$\rightarrow$ activated partial thromboplastin time should be 1.5- to 2 -fold

higher using heparin after normalizing PT-INR (grade C1).

After ICH, antiplatelet treatment has to be individualized, depending on the presence of ischemic vascular diseases or their perceived risk on the one hand and the anticipated risk of ICH recurrence on the other (class IV).

\begin{abstract}
In patients who develop ICH during thrombolytic therapy, thrombolytic drugs and antithrombotic drugs should be immediately stopped, and it is recommended to correct the low levels of coagulation factors such as fibrinogen and prolonged PT and activated partial thromboplastin time using blood products and protamine (grade $\mathrm{C} 1$ ).

Indication for surgical evacuation of the hematoma should be carefully examined while taking account of the functional prognosis after correcting the bleeding tendency (grade C1).
\end{abstract}

After having rechecked the indication for anticoagulation

(following the EUSI recommendations on ischemic stroke), OAT may be continued after 10-14 days, depending on the perceived risk of thromboembolic occlusion and ICH recurrence (class IV).

Table 4. Respiratory management

European guidelines 2006 (EUSI)

Included in the general management and as part of ICP management (see text).
Japanese guidelines 2009

Airway management and/or artificial respiratory management should be considered when disturbance of consciousness has progressed and a respiratory disorder is present during the acute phase (grade $\mathrm{C} 1$ ).

Routine oxygen administration to patients with mild-to-moderate stroke is not recommended (grade C2).

Hyperbaric oxygen therapy for treatment or determination of surgical indication is not recommended (grade C2).
Comparison of Stroke Guidelines: Acute Management of Intracerebral Hemorrhage 
Table 5. Management of brain edema and intracranial hypertension

European guidelines 2006 (EUSI)

Japanese guidelines 2009

The following measurements might be considered in patients

(ICP level is not defined)

with ICH if ICP increases beyond $20 \mathrm{~mm} \mathrm{Hg}$ :

Body position

Elevation of body position with respect to CPP

(CPP >60 mm Hg; class IV, level C).

Raising the upper part of the bed to put the body at a $30^{\circ}$ angle has been reported to be beneficial for patients with intracranial hypertension (grade $\mathrm{C} 1$ ); however, attention should be given to lowering blood pressure.

Osmotic therapy

Glycerol (500 $\mathrm{ml} \mathrm{10 \%} \mathrm{per} \mathrm{day).}$

Mannitol (100 ml 20\%; every 3-6 h; serum osmolality $<320$ $\mathrm{mmol} / \mathrm{l})$.

Hyper-HAES ( $\mathrm{NaCl} 7.5 \%$, HES 6\%, serum sodium $<150 \mathrm{mmol} / \mathrm{l}$ ). THAM buffer (central line because of tissue necrosis) $1 \mathrm{mmol} / \mathrm{kg}$ bolus, $0.25 \mathrm{mmol} / \mathrm{kg}$ as permanent infusion $(\mathrm{pH}<7.5-7.55$;

class IV, level C).
Intravenous administration of hypertonic glycerol is recommended for major acute ICH accompanying intracranial hypertension (grade B).

There is no specific rationale supporting the idea that mannitol treatment is effective for acute ICH (grade C2), but this treatment can be considered when intracranial pressure progressively increases or clinical findings deteriorate in relation to mass effect (grade C1).

In patients using a respirator, a mild hyperventilation ( $\mathrm{pCO}_{2}$ adjusted to $30-35 \mathrm{~mm} \mathrm{Hg}$ ) is recommended (level IIb).

Hyperventilation

Intermittent hyperventilation ( $\mathrm{pCO}_{2}$ level of $30-35 \mathrm{~mm} \mathrm{Hg}$ ), most useful in the first hours (class IV, GCP).

Analgosedation with barbiturates (pentobarbital or thiopental).

Corticosteroids

At the current stage, corticosteroids are not recommended

There is no concrete scientific evidence demonstrating that (class IV, level C).

corticosteroids are effective in reducing acute ICH (grade C2).

Hematoma evacuation with/without craniectomy.

External ventricular drainage in case of hydrocephalus or

clinical deterioration and neuroradiological evidence of

brainstem compression (GCP).

Table 6. Management of seizures

European guidelines 2006 (EUSI)

Early prophylactic treatment of seizures is not recommended for all patients, but may be considered for selected patients with lobar ICH. In all other cases, seizures should only be treated if they occur (level C).

If seizures occur, a stepwise administration of antiepileptic drugs is generally recommended. Antiepileptic treatment should be continued for 30 days. After this time, treatment should be reduced and eventually discontinued. If seizures reoccur, patients should receive chronic treatment with anticonvulsants (level C).
Japanese guidelines 2009

Antiepileptics should be used for seizure attacks in patients with acute stroke. Thereafter, the dosage should be carefully reduced while taking into account the possibility of developing late-onset epilepsy (grade C1).

Seizure occurs frequently in patients with $\mathrm{ICH}$ involving the cerebral cortex. In ICH limited to the putamen, thalamus or infratentorium, concurrent seizure is rare. The prophylactic use of antiepileptics is not recommended except for patients who have undergone brain surgery (grade $\mathrm{C} 2$ ).

In ICH patients who developed late-onset seizures ( $>2$ weeks after onset), recurrent seizures often occur; thus, administration of anti-epileptics is advisable (grade C1). 
Table 7. Prevention of deep venous thrombosis and pulmonary embolism

European guidelines 2006 (EUSI)

Compression stockings and intermittent pneumatic compression are recommended for the prevention of thromboembolism in patients with disabling limb weakness from the beginning of treatment (class IV).

Low-dose subcutaneous heparin or low-molecular-weight heparin should be considered after $24 \mathrm{~h}$, especially in patients who are at high risk of thromboembolism (class IV).
Japanese guidelines 2009

When paralysis is noted in patients with acute $\mathrm{ICH}$, deep venous thrombosis and pulmonary embolisms should be prevented using compression stockings or intermittent pneumatic compression, or the combination of these (grade B).

Administration of low-dose heparin can be considered for ICH patients with concurrent hemiplegia without rebleeding 3-4 days after onset (level IIa).

\section{Respiratory Management}

There is no special recommendation concerning respiratory management for patients with $\mathrm{ICH}$ in the EUSI guidelines (table 4). Oxygenation should be monitored continuously; in addition, respiratory care and prophylactic measures concerning aspiration pneumonia should be part of the general treatment of all stroke patients, including those with ICH. Japanese guidelines give special recommendations for respiratory management.

\section{Management of Brain Edema and Intracranial Hypertension}

EUSI guidelines concerning ICP management give more detailed recommendations, especially for drug dosage and an escalation scheme for treatment (table 5). Both guidelines recommend a mild therapeutic hyperventilation to achieve arterial $\mathrm{pCO}_{2}$ levels of $30-35 \mathrm{~mm}$ $\mathrm{Hg}$, and both recommend elevation of body position at a $30^{\circ}$ angle to decrease ICP. Intravenous administration of hypertonic glycerol is a frequent choice for major acute ICH in Japan. Concerning the application of mannitol, Japanese guidelines recommend the use only in cases of severe mass effect. Barbiturates are not recommended in Japanese guidelines as ultimate treatment option. Both guidelines do not mention hypothermia as therapeutic option to reduce ICP. Japanese guidelines do not recommend hypothermia because of lacking evidence (grade $\mathrm{C} 2$ ). Both guidelines recommend lowering of body temperature in case of fever, using even methods such as cooling.

Comparison of Stroke Guidelines: Acute

Management of Intracerebral Hemorrhage

\section{Management of Seizures}

In Japanese guidelines, the management of early and late-onset seizures is separately recommended. EUSI guidelines are more detailed and recommend a stepwise treatment with recommendations including drug dosages for seizure or status epilepticus treatment (table 6).

\section{Prevention of Deep Venous Thrombosis and Pulmonary Embolism}

Concerning prevention of deep venous thrombosis and pulmonary embolism, both guidelines have similar recommendations (table 7).

\section{Surgical Indication for Acute ICH}

Concerning surgical indication for acute ICH, Japanese guidelines distinguish between treatment in patients with hypertensive ICH and ICH not associated with hypertension. For hypertensive ICH, detailed recommendations according to different locations are provided. EUSI guidelines discern only between supratentorial or infratentorial ICH (tables 8, 9). Supratentorial ICH is divided into superficial (mostly) lobar and deep (mostly basal ganglion) locations, which mainly goes back to the results of STICH (International Surgical Trial in Intracerebral Hemorrhage) [16]. They give no general recommendations for surgical treatment. For infratentorial hemorrhages, good results are mentioned for surgical evacuation of cerebellar hematomas, but optimal timing for surgery is not provided. EUSI guidelines do not give recommendations for brain stem hemorrhage, but they 
Table 8. Surgical indication for supratentorial ICH

European guidelines 2006 (EUSI)

No evidence for a general recommendation (class IV, level C).
Japanese guidelines 2009

General recommendation:

If ICH $<10 \mathrm{ml}$ or mild neurological deficit regardless of the site if $\mathrm{ICH} \rightarrow$ no surgery (grade D).

No rationale for recommending evacuation for patients with deep coma (Japan Coma Scale III-300) (grade C2).

Putaminal hemorrhage:

- Consider evacuation if hematoma volume $>31 \mathrm{ml}$ in patients with moderate neurological findings and evidence of severe mass effect (grade C1).

- Particularly stereotactic evacuation is recommended for patients with disturbed consciousness (Japan Coma Scale II-20 to 30) (grade B).

Thalamic hemorrhage:

No rationale for hematoma evacuation in the acute stage (grade C2).

Stereotactic aspiration may be considered (class IV), especially if mass effect is present.
Ventricular drainage can be considered when accompanied with intraventricular hematoma or marked ventricular dilatation (grade C1).

Lobar (subcortical) hemorrhage:

- Consider surgery if hematoma is $<1 \mathrm{~cm}$ of the surface (grade C1).

- Craniotomy recommended as surgical procedure (grade C1).

Table 9. Surgical indication for infratentorial ICH

European guidelines 2006 (EUSI)

Cerebellar hemorrhage

If cerebellar ICH $>2-3 \mathrm{~cm}$ in diameter, deterioration or brain stem compression and/or hydrocephalus occurs consider surgical evacuation and ventricular drainage as soon as possible (class IV, level C).

\section{Intraventricular hemorrhage}

Intraventricular thrombolysis trials may be considered if an external ventricular drainage becomes necessary (class IV, level C), but not in infants. An intraventricular thrombolysis with urokinase or recombinant tissue plasminogen activator seems to be effective.

\section{Brainstem hemorrhage}

No recommendation.
Japanese guidelines 2009

Surgery can be considered if cerebellar hemorrhage is $>3 \mathrm{~cm}$, if patients deteriorate neurologically, or patients have brain stem compression and hydrocephalus from ventricular obstruction (grade C1).

Because it is quite likely to be associated with vascular disease it is desirable to perform angiography to detect the bleeding source (grade C1).

Ventricular drainage should be considered for acute hydrocephalus (grade $\mathrm{C} 1$ ).

There is no rationale for hematoma evacuation (grade C2). Ventricular drainage can be considered when most of the hematoma is in the ventricle and the ventricle is highly dilated (grade C1).

In case of hydrocephalus External drainage can be ventricular or via the lumbar route if it is a communicating type of hydrocephalus (class IV). Lumbar drainage is definitely contraindicated with all types of obstructive hydrocephalus or if the etiology is in doubt.
Consider external ventricular drainage (grade C1). 
are more detailed concerning treatment of hydrocephalus compared with the Japanese guidelines. Both guidelines recommend the use of external ventricular drainage in case of hydrocephalus.

Organized studies on the selection of treatment procedures are rare. Some trials showed a trend in favor of early surgery in supratentorial nonaneurysmal ICH. The international STICH trial was conducted to demonstrate clinical efficacy of surgery in ICH but was not designed to look at the ideal surgical technique. It suggested a small nonsignificant advantage for surgery. A subgroup analysis showed that patients with superficial hematomas and without IVH presented a more encouraging picture of surgery [16]. Thus we are still unable to specify potential candidates for the optimum surgical technique in current guidelines. The ongoing STICH II trial [17] investigates the hypothesis that patients with superficial lobar hematomas and no IVH might benefit from early hematoma evacuation ( $<12 \mathrm{~h}$ after randomization) [17]. A previously published meta-analysis of 2,186 cases concluded that in particular early surgery ( $<8 \mathrm{~h}$ of ictus) is beneficial [18].

In Japan, a nationwide survey on 7,010 patients with putaminal hemorrhage was conducted, and mortality and functional prognosis 3 months after its onset were compared in 1990 [19]. The survey indicated that surgical therapy for putaminal hemorrhage was beneficial only when it was performed to save the lives of patients in very poor condition. A randomized comparative trial on stereotactic evacuation of putaminal hemorrhage in patients with moderately disturbed consciousness on admission showed a better functional outcome than in those with medical treatment [20].

It has been shown that patients with smaller hemorrhage have a better clinical outcome and a lower mortality, which led to the hypothesis that methods of removing ICH in stable patients could result in a lowered risk of mortality and improved outcome $[21,22]$. The ongoing MISTIE (Minimally Invasive Surgery plus rt-PA for ICH Evacuation) trial [22] (NCT00224770) has the purpose to determine the efficacy of using a combination of minimally invasive surgery and clot lysis with recombinant tissue plasminogen activator to remove ICH using imagebased surgery (MRI or CT) to provide catheter access to ICH for the intervention for clot aspiration followed by instillation of up to 9 doses of recombinant tissue plasminogen activator compared to conventional medical management. The specific objective of MISTIE is to test the efficacy and safety of this intervention and assess its ability to remove blood clots from brain tissue. The treat-

Comparison of Stroke Guidelines: Acute

Management of Intracerebral Hemorrhage ment of spontaneous supratentorial ICH will remain controversial until a definitive prospective randomized controlled trial shows evidence in favor of a particular treatment. At present, the recommendations for or against surgery are based on conflicting evidence.

Also, for IVH, recommendations are difficult due to the lack of evidence. The EUSI recommend intraventricular thrombolysis only within clinical trials, and Japanese recommendations discuss different randomized comparative trials on urokinase treatment, concluding that intraventricular urokinase is effective at least for life-saving treatment for patients with severe IVH [23], but give no recommendation for this therapy. It needs to be mentioned that urokinase might not be available in many countries.

Concerning ICH caused by cerebral arteriovenous malformation, dural arteriovenous fistulas, cavernous angiomas or venous angiomas, Japanese guidelines give very detailed information and recommendations concerning risk factors for bleeding, surgery, embolization, stereotactic radiotherapy, epilepsy and pediatric patients. EUSI guidelines give only a short recommendation for ICH caused by arteriovenous malformations. Japanese recommendations also cover ICH due a to brain tumor ['Surgical treatment is recommended for patients with massive hemorrhage from a brain tumor accompanied by the mass effect (grade C1). Acute deterioration of visual acuity and the visual field due to pituitary apoplexy are indications for emergency surgery (grade C1)'].

\section{Recommendations Mentioned Only in One of the Guidelines}

In the EUSI guidelines, recommendation for brain imaging for ICH diagnosis was described. CT and MRI are equally sensitive, however CT may be faster. ICH in a typical location but at young age or without a history of hypertension needs further diagnostic workup, including MR angiography, CT angiography or digital subtraction angiography to investigate the underlying vascular pathology. However, Japanese guidelines do not refer to imaging strategies.

Japanese guidelines give special recommendations concerning upper gastrointestinal bleeding in patients with hypertensive ICH. Special caution should be exercised to concurrent gastrointestinal bleeding, and prophylactic administration of an antiulcer agent is recommended (C1). EUSI guidelines do not mention upper 
gastrointestinal bleedings. Furthermore, special recommendations for ICH in patients with renal failure are covered only in the Japanese guidelines.

Concerning secondary prevention of depression, European guidelines did not mention this complication, whereas Japanese guidelines recommend the following: Depression frequently occurs after stroke including ICH. It should be assiduously examined for because depression is a factor interfering with cognitive and physical function and activities of daily living (grade B). Drug therapy for a poststroke depressive state is recommended because it is expected to improve depressive symptoms and physical function (grade B).

Both guidelines recommend treatment in specialized care units (in the chapter of Stroke in General in the Japanese guidelines). All patients with acute ICH should preferably be treated in stroke units or in intensive care units if the patient condition requires this, because stroke unit care reduces mortality and increases the likelihood of good functional outcome of stroke in general. Due to a variety of similarities between acute ischemic stroke and $\mathrm{ICH}$, it seems to be plausible that the specialized treatment might also be beneficial for patients with $\mathrm{ICH}(\mathrm{lev}$ el C). Early mobilization and rehabilitation unless intracranial hypertension is present is recommended only in EUSI guidelines (class IV evidence).

\section{Acknowledgments}

We would like to thank Drs. Norio Tanahashi (Saitama Medical University International Medical Center) and Masayasu Matsumoto (Hiroshima University Graduate School of Biomedical and Health Sciences) for their valuable advice.

\section{References}

$>1$ van Asch CJ, Luitse MJ, Rinkel GJ, van der Tweel I, Algra A, Klijn CJ: Incidence, case fatality, and functional outcome of intracerebral haemorrhage over time, according to age, sex, and ethnic origin: a systematic review and meta-analysis. Lancet Neurol 2010;9:167176.

-2 Steiner T, Kaste M, Forsting M, Mendelow D, Kwiecinski H, Szikora I, Juvela S, Marchel A, Chapot R, Cognard C, Unterberg A, Hacke $\mathrm{W}$ : Recommendations for the management of intracranial haemorrhage - part I: spontaneous intracerebral haemorrhage. The European Stroke Initiative Writing Committee and the Writing Committee for the EUSI Executive Committee. Cerebrovasc Dis 2006;22: 294-316.

-3 Shinohara Y, Yanagihara T, Abe K, Yoshimine T, Fujinaka T, Chuma T, Ochi F, Nagayama M, Ogawa A, Suzuki N, Katayama Y, Kimura A, Yasui N: III. Intracerebral hemorrhage. J Stroke Cerebrovasc Dis 2011; 20(suppl 1):S74-S99.

-4 PROGRESS Collaborative Group: Randomised trial of a perindopril-based bloodpressure-lowering regimen among 6,105 individuals with previous stroke or transient ischaemic attack. Lancet 2001;358:10331041.

5 MacMahon S, Peto R, Cutler J, Collins R, Sorlie P, Neaton J, Abbott R, Godwin J, Dyer A, Stamler J: Blood pressure, stroke, and coronary heart disease. Part 1, Prolonged differences in blood pressure: Prospective observational studies corrected for the regression dilution bias. Lancet 1990;335:765-774.
6 Zhang H, Thijs L, Staessen JA: Blood pressure lowering for primary and secondary prevention of stroke. Hypertension 2006;48:187195.

-7 Koga M, Toyoda K, Naganuma M, Kario K, Nakagawara J, Furui E, Shiokawa Y, Hasegawa Y, Okuda S, Yamagami H, Kimura K, Okada Y, Minematsu K, Stroke Acute Management with Urgent Risk-Factor Assessment and Improvement (SAMURAI) Study Investigators: Nationwide survey of antihypertensive treatment for acute intracerebral hemorrhage in Japan. Hypertens Res 2009;32:759-764.

-8 Ohwaki K, Yano E, Nagashima H, Hirata M, Nakagomi T, Tamura A: Blood pressure management in acute intracerebral hemorrhage: relationship between elevated blood pressure and hematoma enlargement. Stroke 2004;35: 1364-1367.

$\checkmark 9$ Itabashi R, Toyoda K, Yasaka M, Kuwashiro T, Nakagaki H, Miyashita F, Okada Y, Naritomi H, Minematsu K: The impact of hyperacute blood pressure lowering on the early clinical outcome following intracerebral hemorrhage. J Hypertens 2008;26:2016-2021.

10 Antihypertensive Treatment of Acute Cerebral Hemorrhage (ATACH) investigators: Antihypertensive treatment of acute cerebral hemorrhage. Crit Care Med 2010;38:637-648.

11 Anderson CS, Huang Y, Wang JG, Arima H, Neal B, Peng B, Heeley E, Skulina C, Parsons MW, Kim JS, Tao QL, Li YC, Jiang JD, Tai LW, Zhang JL, Xu E, Cheng Y, Heritier S, Morgenstern LB, Chalmers J, INTERACT Investigators: Intensive blood pressure reduction in acute cerebral haemorrhage trial (INTERACT): a randomised pilot trial. Lancet Neurol 2008;7:391-399.
12 Morgenstern LB, Hemphill JC 3rd, Anderson C, Becker K, Broderick JP, Connolly ES Jr, Greenberg SM, Huang JN, MacDonald RL, Messé SR, Mitchell PH, Selim M, Tamargo RJ, American Heart Association Stroke Council and Council on Cardiovascular Nursing: Guidelines for the management of spontaneous intracerebral hemorrhage: a guideline for healthcare professionals from the American Heart Association/American Stroke Association. Stroke 2010;41:2108-2129.

13 Delcourt C, Huang Y, Wang J, Heeley E, Lindley R, Stapf C, Tzourio C, Arima H, Parsons M, Sun J, Neal B, Chalmers J, Anderson C, INTERACT2 Investigators: The second (main) phase of an open, randomised, multicentre study to investigate the effectiveness of an intensive blood pressure reduction in acute cerebral haemorrhage trial (INTERACT2). Int J Stroke 2010;5:110-116.

14 Qureshi AI, Palesch YY: Antihypertensive Treatment of Acute Cerebral Hemorrhage (ATACH) II: design, methods, and rationale. Neurocrit Care 2011;15:559-576.

15 Maeda K, Koga M, Okada Y, Kimura K, Yamagami H, Okuda S, Hasegawa Y, Shiokawa Y, Furui E, Nakagawara J, Kario K, Nezu T, Minematsu K, Toyoda K, Stroke Acute Management with Urgent Risk-Factor Assessment and Improvement (SAMURAI) Study Investigators: Nationwide survey of neuro-specialists' opinions on anticoagulant therapy after intracerebral hemorrhage in patients with atrial fibrillation. J Neurol Sci 2012;312:82-85. 
16 Mendelow AD, Gregson BA, Fernandes HM, Murray GD, Teasdale GM, Hope DT, Karimi A, Shaw MD, Barer DH, STICH investigators: Early surgery versus initial conservative treatment in patients with spontaneous supratentorial intracerebral haematomas in the International Surgical Trial in Intracerebral Haemorrhage (STICH): a randomised trial. Lancet 2005;365:387-397.

17 Mendelow AD, Gregson BA, Mitchell PM, Murray GD, Rowan EN, Gholkar AR, STICH II Investigators: Surgical trial in lobar intracerebral haemorrhage (STICH II) protocol. Trials 2011;12:124.
Gregson BA, Broderick JP, Auer LM, Batjer $\mathrm{H}$, Chen XC, Juvela S, Morgenstern LB, Pantazis GC, Teernstra OP, Wang WZ, Zuccarello $\mathrm{M}$, Mendelow $\mathrm{AD}$ : Individual patient data subgroup metaanalysis of surgery for spontaneous supratentorial intracerebral hemorrhage. Stroke 2012;43:1496-1504.

19 Kanaya H: Current situations of treatments of hypertensive intracerebral hemorrhage: results of a national survey (in Japanese). Jpn J Stroke 1990;12:509-524.

20 Hattori N, Katayama Y, Maya Y, Gatherer A: Impact of stereotactic hematoma evacuation on activities of daily living during the chronic period following spontaneous putaminal hemorrhage: a randomized study. J Neurosurg 2004;101:417-420.
21 Lampl Y, Ronit G, Eshel T: Neurological and functional outcome in patients with supratentorial hemorrhages. A prospective study. Stroke 1995;26:2249-2253.

22 Morgan T, Zuccarello M, Narayan R, Keyl P, Lane K, Hanley D: Preliminary findings of the Minimally-Invasive Surgery plus rtPA for Intracerebral Hemorrhage Evacuation (MISTIE) clinical trial. Acta Neurochir Suppl 2008;105:147-151.

23 Andrews CO, Engelhard HH: Fibrinolytic therapy in intraventricular hemorrhage. Ann Pharmacother 2001;35:1435-1448. 